TAUP 2674-2001

\title{
The Effect of a Magnetic Flux Line in Quantum Theory
}

\author{
Y. Aharonov ${ }^{(a, b)}$, T. Kaufherr ${ }^{(a) 1}$ \\ (a) School of Physics and Astronomy, Tel Aviv University, Tel Aviv 69978, Israel \\ (b)Department of Physics, University of South Carolina, Columbia, S.C. 29208
}

\begin{abstract}
The nonloclal exchange of the conserved, gauge invariant quantity $e^{\frac{i}{\hbar}\left(p_{k}-\frac{e}{c} A_{k}\right) L^{k}}, L^{k}=$ const., $k=1,2$ between the charged particle and the magnetic flux line (in the $k=3$ direction), is responsible for the AharonovBohm effect. This exchange occurs at a definite time, before the wavepackets are brought together to interfere, and can be verified experimentally.
\end{abstract}

PACS numbers: 03.65.Vf, 03.65.Ta, 03.65.Ud

${ }^{1}$ email address trka@post.tau.ac.il 


\section{Introduction}

The Aharonov-Bohm (A-B) effect [1] is usually described in terms of the shift in the interference pattern created by a charged particle moving outside a solenoid. This description leaves open the question regarding the time when the effect occurs. Since the shift is due to a change in the relative phase between the two wave packets of the particle, it seemed natural to look for an answer in the time evolution of the relative phase. But the relative phase is gauge dependent; it is only when the two wave packets meet that it becomes gauge invariant. Thus it seems that quantum theory has contrived to eliminate any trace of when the effect occurs, and that gauge invariance has some part in this.

Below we show that the relevant variables are the gauge invariant displacement operators $e^{\frac{i}{\hbar}\left(p_{k}-\frac{e}{c} A_{k}\right) L^{k}}, \quad L^{k}=$ const., $k=1,2$ for a flux line in the $k=3$ direction (secII). In the A-B setup, these otherwise conserved quantities are exchanged nonlocally and at a definite time, between the charged particle and the flux line (secsIII,IV). This accounts for the A-B effect (secsII,III). The exchange alters the velocity distribution of the charged particle and can therefore be verified experimentally (secIII).

\section{Modular Momentum}

Quantum interference, unlike classical interference ${ }^{1}$, is a manifestation of an underlying dynamics. To see this, consider a setup consisting of a charged particle moving in the vicinity of a parallel, infinite plate capacitor, but prevented from entering the electric field. Consider first the position and momentum of the particle. The Heisenberg equations of motion are,

$$
\begin{aligned}
& \dot{\vec{x}}=\frac{i}{\hbar}[H, \vec{x}]=\frac{\vec{p}}{m}, \\
& \dot{\vec{p}}=\frac{i}{\hbar}[H, \vec{p}]=e \vec{E} .
\end{aligned}
$$

Since the electric field vanishes in the region where the particle can be found, the average position and momentum of the particle are unaffected by the electric field. Also the averages of higher moments of position and momentum remain unaffected. So far the situation in quantum theory is similar to the classical one. But consider the displacement operator

$$
\begin{gathered}
f\left(p_{x}\right)=e^{\frac{i}{\hbar} p_{x} L} . \\
\frac{d f}{d t}=\frac{i}{\hbar}[H, f]=\frac{i e}{\hbar}\left(\int_{x}^{x+L} E d x^{\prime}\right) f
\end{gathered}
$$


where $x$ denotes the position of the particle. We have thus discovered a quantity that changes. Compare this with the classical analog of (3),

$$
f\left(p_{x}\right)=e^{i 2 \pi \frac{p_{x}}{p_{0}}}
$$

where $p_{0}=\frac{h}{L}$. It satisfies

$$
\frac{d f\left(p_{x}\right)}{d t}=-\left\{H, f\left(p_{x}\right)\right\}=e E \frac{\partial f}{\partial p_{x}}=i \frac{2 \pi}{p_{0}} e E f,
$$

where \{\} denote Poisson brackets. It too does not change unless the particle actually comes in contact with the field. The displacement operator $f\left(p_{x}\right)$ is a function of modular momentum $p_{x}\left(\bmod p_{0}\right)[2,3]$ defined by

$$
p_{x}\left(\bmod p_{0}\right)=p_{x}-N p_{0}
$$

and so that its eigenvalues satisfy

$$
0 \leq p_{x}\left(\bmod p_{0}\right) \leq p_{0}
$$

$N$ is an operator having integer eigenvalues. It is instructive to consider the displacement operator also in the Schrödinger representation. Let the state of the particle be

$$
\Psi_{\alpha}=\Psi_{1}+e^{i \alpha} \Psi_{2},
$$

where $\Psi_{1}$ and $\Psi_{2}$ are non-overlapping wavepackets, and $\Psi_{2}(x)=\Psi_{1}(x-L)$. $\alpha$ represents the change in the wavefunction of the charged particle due to its interaction with the capacitor. The average values of $x$ and $p_{x}$ do not depend on $\alpha$ nor do averages of any polynomial in $x$ or $p_{x}$. Yet $^{2}$

$$
\left\langle e^{i 2 \pi \frac{p_{x}}{p_{0}}}\right\rangle=\frac{1}{2} e^{i \alpha}
$$

The implication of this is that the modular momentum has changed. compare this with the interference of classical waves. Consider as an example two nonoverlapping wavepackets of classical electromagnetic radiation. Introducing a relative phase between the two will not change the total energy and momentum $\frac{1}{8 \pi} \int\left(\mathcal{E}^{2}+B^{2}\right) d v, \frac{1}{4 \pi c} \int(\overrightarrow{\mathcal{E}} \times \vec{B}) d v$, of the field. This of course continues to be true even when the two wavepackets come to overlap, and a shift in the interference pattern due to the change in relative phase is observed. When the electromagnetic field is quantized, the operator $e^{\frac{i}{\hbar} \frac{1}{4 \pi c} \int(\overrightarrow{\mathcal{E}} \times \vec{B}) d v \cdot \vec{L}}=e^{\frac{i}{\hbar} \vec{P} \cdot \vec{L}}$, where $\vec{P}$ is the total momentum, acts as a displacement operator on every state of the field. Thus, for a single photon in a superposition of two wavepackets displaced by $\vec{L}$, this operator depends on the relative phase in exactly the same way as the modular momentum considered above does. 
Modular momentum has its own conservation law. Consider a collision between two systems 1 and 2 such as the charged particle and capacitor, and let

$$
\begin{aligned}
& \pi_{1}=\cos \left(2 \pi \frac{p_{1}}{p_{0}}\right) \\
& \pi_{2}=\cos \left(2 \pi \frac{p_{2}}{p_{0}}\right),
\end{aligned}
$$

where $p_{1}, p_{2}$ are the components of the momentum in any given direction. Conservation of momentum in that direction, i.e.,

$$
p_{1}+p_{2}=p_{1}^{\prime}+p_{2}^{\prime}
$$

then implies that also $C=\cos 2 \pi \frac{p_{1}^{\prime}+p_{2}^{\prime}}{p_{0}}$ is conserved, which leads to the conservation law (see fig.1)

$$
1-C^{2}=\left(\pi_{1}^{\prime}\right)^{2}+\left(\pi_{2}^{\prime}\right)^{2}-2 C \pi_{1}^{\prime} \pi_{2}^{\prime},
$$

which, in general, describes an ellipse. During a collision, modular momentum is exchanged under the constraint (14). Obviously, $\Delta \pi_{2}$ need not in general be equal to $-\Delta \pi_{1}$ (where $\Delta \pi_{1}=\pi_{1}^{\prime}-\pi_{1}, \Delta \pi_{2}=\pi_{2}^{\prime}-\pi_{2}$. .) Instead, the systems translate from one point of the ellipse to another.

Finally, we show that a change in modular momentum, occurring while the wavepackets are separated, manifests itself later as a shift in the interference pattern. Consider the case where, after the capacitor is closed, the two packets are allowed to meet. Then,

$$
\Psi_{\alpha}(x, 0)=\Psi_{1} e^{i k_{0} x}+e^{i \alpha} \Psi_{2} e^{-i k_{0} x} .
$$

In this case, the average of

$$
O(0)=\cos \left[\frac{1}{\hbar} p_{x}(0) L+2 k_{0} x(0)\right]
$$

which is a function of modular momentum, depends on $\alpha$. We use the Heisenberg representation. Since $p_{x}(0)=p_{x}(t) \equiv p_{x}$ and $x(0)=x(t)-\frac{p_{x}}{m} t$ are constants of the motion,

$$
=\cos \left[\frac{1}{\hbar} p_{x} L+2 k_{0}\left(x(t)-\frac{p_{x}}{m} t\right)\right] \equiv O(t),
$$

and

$$
<O(t)>\equiv \frac{1}{2} \cos \alpha .
$$

In particular, at $T=\frac{m L}{2 \hbar k_{0}} O$ turns into the local function $O(T)=\cos \left[2 k_{0} x(t)\right]$, which signals interference. $\langle O(T)\rangle=\frac{1}{2} \cos \alpha$ is then a sufficient condition for the shift of the interference pattern.

\section{The effect of the vector potential on the velocity distribution}

With the aid of the gauge invariant version of modular variables it is possible to determine the time when the A-B effect occurs. Consider a charged 
particle prepared in a superposition of two wavepackets

$$
\Psi=\Psi_{1}+\Psi_{2}
$$

where $\Psi_{2}(y)=\Psi_{1}(y-L), L \gg \Delta y, \Delta y$ is the spread of the packets. The particle passes by a thin solenoid enclosing a flux $\Phi$, but sufficiently far from it, so that its overlap with the solenoid is negligible. Thus it always moves in the field free region surrounding the solenoid. We want to show that the distribution of the velocity in the direction of the line connecting the two wavepackets changes when this line crosses the solenoid provided the solenoid lies in the interval between the packets (see fig. 2). Without loss of generality we can assume that the line connecting the two packets is in the $y$ direction. With the magnetic field within the solenoid pointing in the $+z$ direction, let us choose the gauge

$$
A_{x}=-\Phi \delta(x) \theta(y), \quad A_{y} \equiv 0,
$$

where $\theta(y)$ is the step function. We choose this gauge so that before and after the particle passes by the solenoid $\vec{p}=m \vec{v}$, since the vector potential vanishes in that region. The Fourier transform of the velocity distribution is then (we take $\hbar=1$ )

$$
\int P_{r}\left(m v_{y}\right) e^{i m v_{y} L} d v_{y}=\int P_{r}\left(p_{y}\right) e^{i p_{y} L} d p_{y}=<\Psi\left|e^{i p_{y} L}\right| \Psi>=\frac{1}{2} .
$$

After $\Psi_{2}$ has crossed the potential line, the particle's state is given by

$$
\Psi^{\prime}=\Psi_{1}+e^{-i \alpha} \Psi_{2},
$$

where $\alpha=\frac{e \Phi}{c \hbar}$. Consequently, the Fourier transform of the velocity distribution has changed by

$$
\delta\left\langle e^{i m v_{y} L}\right\rangle=\frac{1}{2}\left(e^{-i \alpha}-1\right) .
$$

It is instructive to consider the situation in the Coulomb gauge where $\vec{A}=\frac{\Phi}{2 \pi r} \hat{e}_{\varphi}$. Comparing the value of the Fourier transform of the velocity distribution shortly before the particle has passed by the solenoid, with its value immediately afterwards, we find that it has changed by ${ }^{3}$

$$
\delta\left\langle e^{i m \vec{v} \cdot \vec{L}}\right\rangle=\frac{1}{2}\left(e^{-i \frac{e}{c} \oint \vec{A} \cdot d \vec{l}}-1\right)=\frac{1}{2}\left(e^{-i \frac{e}{c} \Phi}-1\right),
$$

in agreement with (23), having used Stokes's theorem and

$$
e^{i\left(\vec{p}-\frac{e}{c} \vec{A}\right) \cdot \vec{L}}=e^{i \frac{e}{c} \int_{\vec{r}+\vec{L}}^{\vec{A}} \vec{A} \cdot d \vec{l}} e^{i \vec{p} \cdot \vec{L}}
$$

Thus, the change in the Fourier transform of the velocity distribution occurs when the line connecting the wave packets crosses the solenoid, even though in this gauge there is no sudden change in the relative phase between the 
packets. To test this experimentally, we suggest to measure, at a given time $\mathrm{t}$, the velocity distribution of an ensemble of $\mathrm{N}$ charged particles prepared in a state $\Psi$ such as (19), and to calculate its Fourier transform. Repeating this procedure, using different ensembles, at a succession of times during the motion of the charged particle towards the flux line and past it, should verify the existence of the effect.

(23) causes the shift in the interference pattern. This follows by applying the argument from the end of sec II to ${ }^{4} O(t)=\cos \left[m \dot{y} L+2 k_{0}(y-\dot{y} t)\right]$. Thus it is the gauge invariant exchange, occurring while the charged particle passes by the solenoid, and not any subsequent change in the relative phase (which is not gauge invariant) that is responsible for the shift in the interference pattern.

\section{The effect of the vector potential on the angular velocity dis- tribution}

The exchange of modular angular velocity occurs on a circle. To demonstrate this, we refer to the same setup as before, i.e., a charged particle in a superposition of two wavepackets moving towards a solenoid. For the sake of simplicity we shall consider this case in the reference frame where the center of mass of the charged particle is at rest. We define the coordinate system thus: the origin coincides with the center of mass of the charged particle, the $y$ axis passes through the centers of the two wavepackets. The state of the charged particle is given by

$$
\Psi=\Psi_{1}+\Psi_{2}
$$

where $\Psi_{2}(\varphi, r)=\Psi_{1}(\varphi-\pi, r) . \quad \Psi_{1}$ and $\Psi_{2}$ are two nonoverlapping wave packets that are at rest, $\mathrm{r}$ is the distance of their centers from the origin (see fig. 3). The flux enclosed by the solenoid is $\Phi$, with the magnetic field pointing in the $+z$ direction. The solenoid moves towards the particle in the $-x$ direction. The modular angular velocity about the z-axis is given by

$$
e^{i I v_{\varphi} \pi}=e^{i\left(p_{\varphi}-\frac{e}{c} r A_{\varphi}\right) \pi}=e^{i \frac{e}{c} \int_{\varphi^{\prime}=\varphi+\pi, r^{\prime} \equiv r}^{\varphi} \vec{A} \cdot \overrightarrow{d l}^{\prime}} e^{i p_{\varphi}},
$$

having used relation (25) $\cdot p_{\varphi}$ and $I$ are, respectively, the angular momentum and moment of inertia about the $z$ axis. The expectation value equals

$$
\left\langle e^{i I v_{\varphi} \pi}\right\rangle=1 \rightarrow \frac{1+e^{-i \alpha}}{2} \rightarrow \cos \alpha
$$

where $\alpha=\frac{e \Phi}{\hbar c}$. As the solenoid moves toward the charged particle and then away from it, the average angular modular velocity changes abruptly twice, on the solenoid's crossing the circle of radius $r$ centered at the origin. This, too, is an experimentally verifiable result. 


\section{Discussion}

The commonly accepted view is that, in order to measure the A-B effect, two wavepackets moving on the two sides of a solenoid have to be brought together to interfere. This also epitomizes the topological nature of the effect. The picture behind this is that of the relative phase being collected gradually while the wavepackets travel through the nonsimply connected region surrounding the solenoid, from which the potentials can never be completely eliminated. We have shown that this is not so, that the effect can be experimentally ascertained to occur at an earlier stage, when the wavepackets are still separated. The effect results from a nonlocal exchange of a conserved, gauge invariant modular quantity between the charged particle and the solenoid, which occurs at a definite time. It is somewhat disturbing that this quantity contains information about a region which the particle has never traversed ${ }^{5}$. For example, in the context of section III, in the gauge $A_{x} \equiv 0, A_{y}=\Phi \theta(x) \delta(y)$. Note nevertheless that the measurement of velocity necessarily brings the particle to the region of that part of the vector potential that has not yet affected the phase of the wavefunction. But we also know that the measurement gives the distribution of the velocities as it existed before the measurement.

This research was supported in part by Grant No.62/01 of the Israel Science Foundation, by the NSF Grant No. NSF 0140377, and by the ONR Grant No. N00014-00-0383. T. K. acknowledges the support of the Grant-in-Aid for Specially Promoted Research, No. 10101001, by Monbusho, Japan. Special thanks to Prof. H. Yoshiki of Kure University, Hiroshima, who led this grant. 


\section{Footnotes}

1. Below, we shall consider the interference of classical electromagnetic waves and also its quantum counterpart.

2. Note that because $\Psi_{\alpha}$ is a non-analytic function of $x$,

$$
\left\langle e^{i 2 \pi \frac{p_{x}}{p_{0}}}\right\rangle=\left\langle\sum_{n} \frac{(2 \pi i)^{n}}{n !}\left(\frac{p_{x}}{p_{0}}\right)^{n}\right\rangle \neq \sum_{n} \frac{(2 \pi i)^{n}}{n !}\left\langle\left(\frac{p_{x}}{p_{0}}\right)^{n}\right\rangle .
$$

3. In the Coulomb gauge,

$$
A_{x}=\frac{\Phi}{2 \pi}\left(-\frac{y}{r^{2}}\right), \quad A_{y}=\frac{\Phi}{2 \pi}\left(\frac{x}{r^{2}}\right) .
$$

At $x=-\varepsilon$, the state of the particle is

$$
\Psi=\Psi_{1}+e^{i \varphi_{0}} \Psi_{2}=\psi\left(y-\frac{L}{2}\right)+e^{i \varphi_{0}} \psi\left(y+\frac{L}{2}\right),
$$

where we assume that $\psi(y)$ is centered about the origin.

$$
\varphi_{0}=\frac{e}{c} \int_{\frac{L}{2}}^{-\frac{L}{2}} A_{y}\left(y^{\prime}, x=-\varepsilon\right) d y^{\prime} .
$$

At $x=+\varepsilon$, the state of the particle is

$$
\Psi^{\prime}=\Psi_{1} e^{i S_{1}}+e^{i \varphi_{0}} \Psi_{2} e^{i S_{2}},
$$

where

$$
\begin{aligned}
& S_{1}=\frac{e}{c} \int_{-\varepsilon}^{\varepsilon} A_{x}\left(x^{\prime}, y=\frac{L}{2}\right) d x^{\prime}, \\
& S_{2}=\frac{e}{c} \int_{-\varepsilon}^{\varepsilon} A_{x}\left(x^{\prime}, y=-\frac{L}{2}\right) d x^{\prime} \stackrel{30}{=}-S_{1} .
\end{aligned}
$$

We have

$$
<\Psi\left|e^{i m v_{y} L}\right| \Psi>=\frac{1}{2},
$$

and

$$
\begin{aligned}
& <\Psi^{\prime}\left|e^{i m v_{y} L}\right| \Psi^{\prime}>=\int d y \Psi^{\prime *}(y) e^{-i \frac{e}{c} \int_{y}^{y+L} A_{y}\left(y^{\prime}, x=\varepsilon\right) d y^{\prime}} e^{i p_{y} L} \Psi^{\prime}(y) \\
\approx & e^{-i \varphi_{0}} e^{i S_{1}} e^{-i S_{2}} e^{-i \frac{e}{c} \int_{-\frac{L}{2}}^{\frac{L}{2}} A_{y}(y, x=\varepsilon) d y} \int d y \psi^{*}\left(y+\frac{L}{2}\right) \psi\left(y+\frac{L}{2}\right) \\
= & \frac{1}{2} e^{-i \frac{e}{c} \oint \vec{A} \cdot d \vec{l}}=\frac{1}{2} e^{-i \frac{e}{c} \Phi},
\end{aligned}
$$


having used (25), (32), (34), (35) and Stokes's theorem. We finally obtain,

$$
\delta\left\langle e^{i m \vec{v} \cdot \vec{L}}\right\rangle=<\Psi^{\prime}\left|e^{i m v_{y} L}\right| \Psi^{\prime}>-<\Psi\left|e^{i m v_{y} L}\right| \Psi>=\frac{1}{2}\left(e^{-i \frac{e}{c} \Phi}-1\right) .
$$

4. Here expressed in the Schrödinger representation.

5. In this connection, see also [4].

\section{References:}

[1] Y. Aharonov and D. Bohm, Phys. Rev. 115 (1959) 485.

[2] Y. Aharonov, H. Pendelton and A. Petersen, Int. J. Th. Phys. 2 (1969) 213.

[3] Y. Aharonov, Proc. Int. Sym. Foundations of Quantum Mechanics in the Light of New Technology, Ed. S. Kamefuchi et. al. Phys. Soc. Japan,(1984) 10.

[4] M. V. Berry, Proc. R. Soc. Lond. A392, (1984) 45. 


\section{Captions for Figures:}

Fig. 1. Conservation law of modular momentum

$$
\begin{aligned}
& \pi_{1}^{\prime}=\cos \left(2 \pi \frac{p_{1}^{\prime}}{p_{0}}\right) \\
& \pi_{2}^{\prime}=\cos \left(2 \pi \frac{p_{2}^{\prime}}{p_{0}}\right)
\end{aligned}
$$

$p_{0}$ is a constant. $p_{1}^{\prime}, p_{2}^{\prime}$ are the components of the momentum in any given direction after a collision. Due to a collision, the system moves from one point of the ellipse to another. For example, from a to b.

Fig. 2. Charged particle in a superposition of three wavepackets moving in the $\mathrm{x}$-direction. Only the distributions of the velocities in the directions $\mathrm{AB}$ and $\mathrm{AC}$ will change when the respective lines cross the solenoid, while the distribution of the velocity in the direction $\mathrm{BC}$ does not change during the motion.

Fig. 3. View from the reference frame where the center of mass of the charged particle is at rest: As the solenoid crosses the circle line of radius $r$, the modular angular velocity about the $z$ axis of the charged particle changes. 


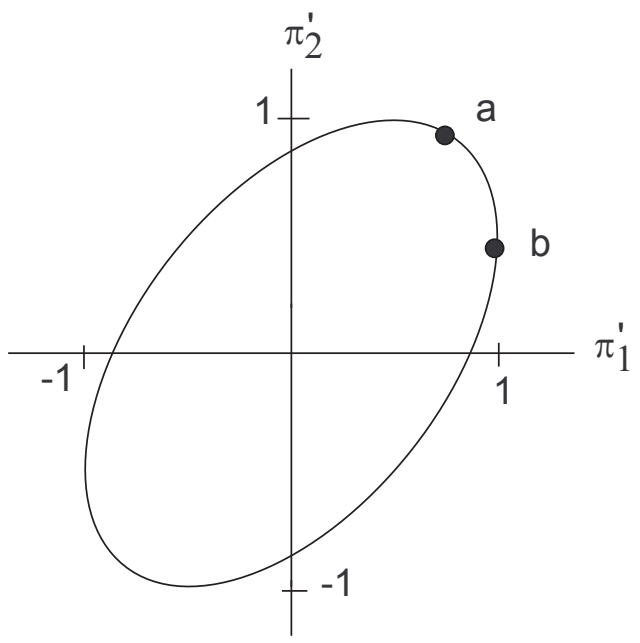

11 


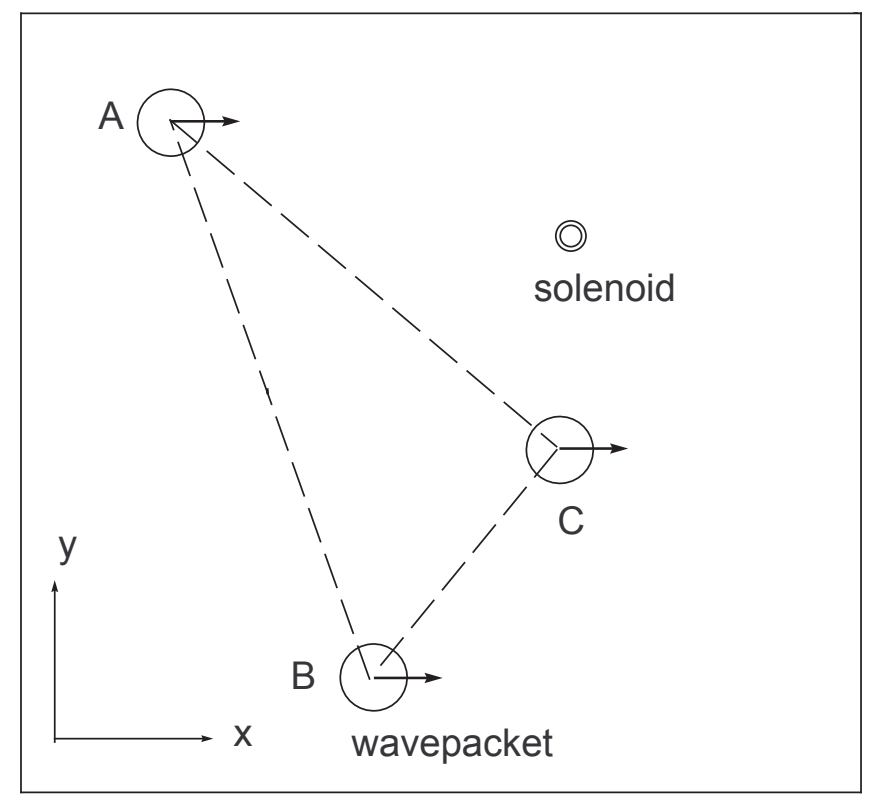




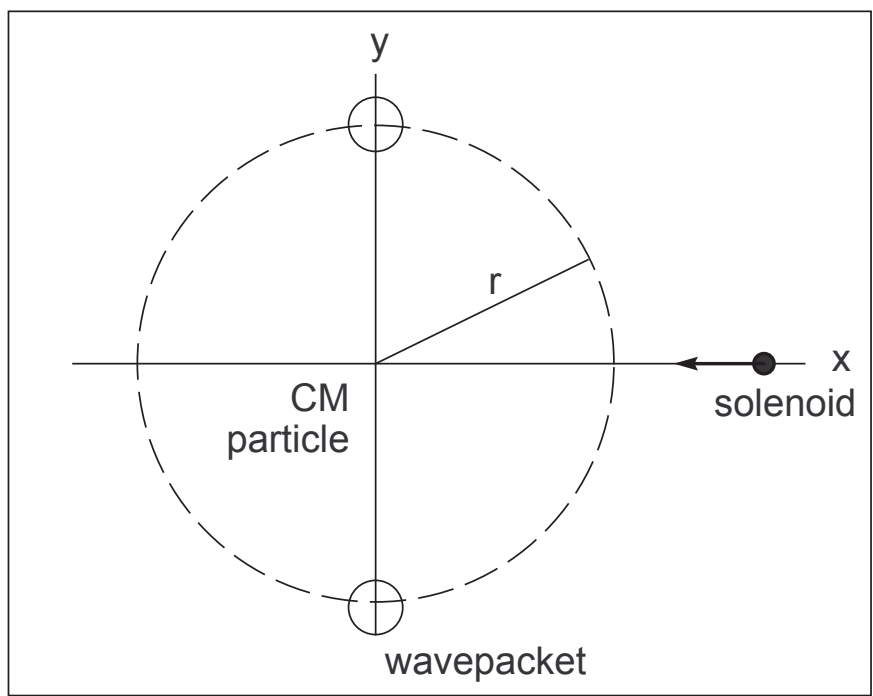

